\title{
Generic Communication Framework for Internet of Things
}

\author{
Amol Dande ${ }^{1}$, S. K. Pathan ${ }^{2}$ \\ ${ }^{1}$ Smt. Kashibai Navale College of Engineering, Pune, Maharashtra, India \\ ${ }^{2}$ Professor, Smt. Kashibai Navale College of Engineering, Pune, Maharashtra, India
}

\begin{abstract}
Internet of Things the name explains about itself, internet is network of network where heterogeneous machine are connected to share the information among different clients. Things are the sensors connected to this network. Network layer and physical layer wrapped together with specific application layer to form IoT, which becomes domain specific .There are various frameworks exists which are dependent on specific domain, if new domain comes, ultimately there is need to create new framework. In this paper we proposed generic communication framework which is common platform for all the domains, where IoT can be used and existing framework can be reused. It is beneficial to analyze data efficiently and future prediction.
\end{abstract}

Keywords: Internet of Things, IoT solutions, email communication, data storage and analysis, machine to machine communication, cloud structure for IoT.

\section{Introduction}

IoT network infrastructure consists of multiple devices (sensors) are connected using communication protocols. IoT is $n / w$ of $n / w$ anything can be accessed from anywhere via various application programming interface. In IoT there is a continuity to development, future scope can be esti mated by a mixture of variety of technology path and various IT concepts which consist of CC, Hadoop, Robot and other different fields. The concept is came from ubiquitous computing, so these ideas lap in few component can be service infrastructure ,technical, visualization, ability of system to work with other system, self-decision, actual innovators can see great the vision of complementary instead against separate fields. The inference of concept IoT can be globally defined as the completeness of presence things, an IoT area of world may be connected to object everywhere on the planet. As like as computer network and the ubiquitous computing works as per the human body, such as human neurons are capable for taking the decision simultaneously likewise this way or path can be used in IoT for higher utilization and fast decision making. Devices deeply embedded in public and private places will recognize us and adapt to our requirements for comfort, safety, streamlined commerce, entertainment, education, resource conservation, operational efficiency and personal well-being.", according to Intel's report "Rise of the Embedded Internet [1].Four companies are emerging as IoT leaders: Intel in the semiconductor space, IBM and Microsoft in the platform/analytic s space and Cisco in the connectivity.In addition, designate one author as the "corresponding author". This is the author to whom proofs of the paper will be sent. Proofs are sent to the corresponding author only [2].

\section{Related work}

There exist various [3] application under dif- ferent domains in Internet of Things. Internet of things is application driven which tends to new innovation, most common domains are
Smart wear, Transportation, Home-appliances, information of environment, and human Care. And some of the challenges are in the sys- tem of one technology get communicates with other technology made system architecture , Privacy and Security preserving, smart physical object(things), realistic and flexible. Al- most all the applications are domain specifics. One of the applications designed to human safety.

\section{A. Industries towards the IoT}

IoT is described as the broad idea behind these buzzwords is that a whole constellation of inanimate objects is being designed with built-in wireless connectivity, so that they can be monitored, controlled and linked over the Internet via a mobile app. [4] The types of objects span a wide range of categories, from wearables to light bulbs to home appliances (like the coffee maker, washing machine, and Even your car) really, anything. [5]IoT is also being applied to vertical markets like the medical and health-care industry and to transportation systems. At this point, the easier question might be who isn't working on an IoT product.[6] Big names like Samsung, LG, Apple, Google, Lowe's and Philips are all working on connected devices, as are many smaller companies and start-ups.[7] Research group Gartner predicts that 4.9 billion connected devices will be in use this year, and the number will reach 25 billion by 2020 .

\section{B. IoT Controllers and hardware's:}

There are various micro controllers and development boards are available to implementing the IoT. Some of the controllers list is given below. 


\section{International Journal of Science and Research (IJSR) \\ ISSN (Online): 2319-7064}

Index Copernicus Value (2013): 6.14 | Impact Factor (2015): 6.391

\begin{tabular}{|c|c|c|}
\hline \multicolumn{3}{|r|}{ IoT Hardware } \\
\hline No & H/W & Summary \\
\hline 1 & $\begin{array}{c}\text { Raspberry-pi } \\
2\end{array}$ & $\begin{array}{l}\text { The Open source, Broadcom BCM2835 } \\
\text { 700MHz processor, GPIO: } 8 \text { pins, SD Card } \\
\text { socket, HDMI and connectivity via Ethernet }\end{array}$ \\
\hline 2 & $\begin{array}{l}\text { Microsoft } \\
\text { IoT pack for } \\
\text { Raspberry-pi }\end{array}$ & $\begin{array}{l}\text { Open source device and gets you started with } \\
\text { Win } 10 \text { Core, ARM9 } 200 \mathrm{MHz} \text { processor, } 8 \\
\text { digital inputs, } 2 \text { analogue inputs, } 2 \text { digital } \\
\text { outputs, } 2 \text { serial ports RS-232, } 1 \text { serial port } \\
\text { RS-485 and built-in web server. Connectivity } \\
\text { via Ethernet. }\end{array}$ \\
\hline 3 & $\begin{array}{l}\text { Arduino- } \\
\text { Uno }\end{array}$ & $\begin{array}{l}\text { Open source, ATmega328 microcontroller, } \\
14 \text { digital and } 6 \text { analogue } \mathrm{I} / \mathrm{O} \text { pins, } 32 \mathrm{k} \text { Flash } \\
\text { Memory and Internet connectivity via Shields }\end{array}$ \\
\hline 4 & $\begin{array}{l}\text { Beagle } \\
\text { Bone }\end{array}$ & $\begin{array}{l}\text { Open source, AM335x 1GHz ARM }{ }^{\circledR} \\
\text { Cortex-A8, } 2 \text { GB of on-board flash, a } \\
\text { microSD card reader, HDMI, GPIO } 65 \text { pins, } \\
\text { and connectivity via Ethernet. }\end{array}$ \\
\hline 5 & $\begin{array}{c}\text { Wunder } \\
\text { Bar }\end{array}$ & $\begin{array}{l}\text { Open source SDK, Main circuit: NXP } \\
\text { LPC1837 Cortex M3, connectivity via WiFi } \\
\text { BTLEAnd } 6 \text { sensor circuits with Cortex M0 } \\
\text { processor and connectivity via BTLE: } \\
\text { light/colour/proximity, Gyroscope / } \\
\text { Accelerometer, Thermometer / Humidity, IR } \\
\text { Transmitter (remote control), Sound and } \\
\text { Bridge/Grove with } 2 \text { GPIO pins }\end{array}$ \\
\hline 6 & $\begin{array}{l}\text { IoT } \\
\text { Develpo- } \\
\text { Ment } \\
\text { Platform }\end{array}$ & $\begin{array}{c}\text { Oracle Java ME environment, Customised } \\
\text { ARM-based processor, 3G/2G mobile } \\
\text { connectivity, WiFi, GPS, Oracle JavaME } \\
\text { Embedded SDK, Low Level Device I/O } \\
\text { Access, AC adapter and Battery with onboard } \\
\text { charging }\end{array}$ \\
\hline 7 & ARMmbed & $\begin{array}{c}\text { For IBM cloud environment, ARM } \AA \\
\text { Cortex }{ }^{\mathrm{TM}}-\mathrm{M} 4 \text { Core, } 128 \times 32 \text { Graphics LCD, } \\
\text { 5-way joystick, } 2 \text { x Potentiometers, speaker, } \\
\text { accelerometer and temperature sensor }\end{array}$ \\
\hline 8 & Waspmote & $\begin{array}{l}\text { Open source, ATmega1281, Over-The-Air } \\
\text { programming, } 60 \text { sensors available to } \\
\text { connect to Waspmote, On-Board } \\
\text { Temperature and Accelerometer, Hibernate } \\
\text { mode consumes just } 0.06 \mu \mathrm{A} \text {, connectivity: } \\
\text { 3G/GPRS, WiFi, BT }\end{array}$ \\
\hline 9 & $\begin{array}{c}\text { ThingsEE } \\
\text { One }\end{array}$ & $\begin{array}{l}\text { Open source, ARM® based Cortex®-M3, } 2 \mathrm{G} \\
\text { / WiFi connectivity, accelerometer, } \\
\text { temperature/humidity, GPS, orientation with } \\
\text { 9-axis inertial module, ambient light sensor, } \\
\text { SD card slot and one-year-battery-life with } \\
\text { 1900mAh battery (charge via mUSB). }\end{array}$ \\
\hline
\end{tabular}

Internet of Things has moved on from high-level hype towards concrete ideas and products, and a growing business developer community is eagerly looking for either suitable hardware for rapid prototyping or devices with which to deploy their ideas. We thought we'd compile a list of some of the more prominent hardware options on the market [15]

\section{Cloud Structure for IoT:}

Now you can harness the power of the Internet of Things and turn the data generated by every one of your customers, partners, devices, and sensors into meaningful action. [9]With IoT Cloud you can process massive quantities of data, build business rules with simple, intuitive tools, and engage proactively with customers in real time [10]. [11] The
Bosch IoT Suite provides the foundation for service enablement, both in terms of connecting things to the Internet - reliably, securely, cost effectively and at scale - and in terms of delivering the backing application logic for valueadded services. It is made up of a set of software services that provide all of key middleware capabilities needed to build a sophisticated IoT application from top to bottom. Customers can use any combination of these IoT services as needed to rapidly implement the desired solution.IoT is vast, diverse and constantly changing. IoT data from billions of interactions between devices and people is not only massive, it is complex and variable. Predefined programs aren't up to the task of analysing it. And traditional systems can't make sense all IoT data combined with unstructured data, like weather and social.A cognitive IoT can make sense of all types of data. In fact, it can choose its own data sources and decide which patterns and relationships to pay attention to. It uses machine learning and advanced processing to organize the data and generate insights. A cognitive IoT can also evolve and improve on its own through learned selfcorrection and adaptation. IBM released Watson cloudplatform for Internet of Things [12].

[13] The AWS IoT Device SDK helps you to easily and quickly connect your hardware device to AWS IoT. It provides enhanced features so that your hardware device can seamlessly and securely work with the device gateway and device shadow provided by AWS IoT. The AWS IoT Device SDK includes open source libraries, the developer guide with samples, and the porting guide so that you can build innovative IoT products or solutions on your choice of hardware platforms.[14] Cisco Internet of Things (IoT) Cloud Connect is our new mobility-cloud-based software suite. It offers a complete solution for mobile operators to provide exceptional IoT experiences. Cisco IoT Cloud Connect helps you find new ways to make money, while fully optimizing and utilizing your networks. Cisco provides granular, real-time visibility and updates across every level of the network - transport and user, access, core, and cloud.

\section{Comparison with similar system}

Table

\begin{tabular}{|c|c|c|}
\hline Parameter/System & Existing System & Proposed Work \\
\hline Automation & Yes & Yes \\
\hline Application Domain & Domain Specific & Generic \\
\hline Manual Control & No & Yes \\
\hline Access Devices via E-mail & No & Yes \\
\hline $\begin{array}{c}\text { Integration Of Multiple IoT } \\
\text { Application }\end{array}$ & No & Yes \\
\hline Text Notification & Yes & Yes \\
\hline
\end{tabular}

\section{Proposed Work}




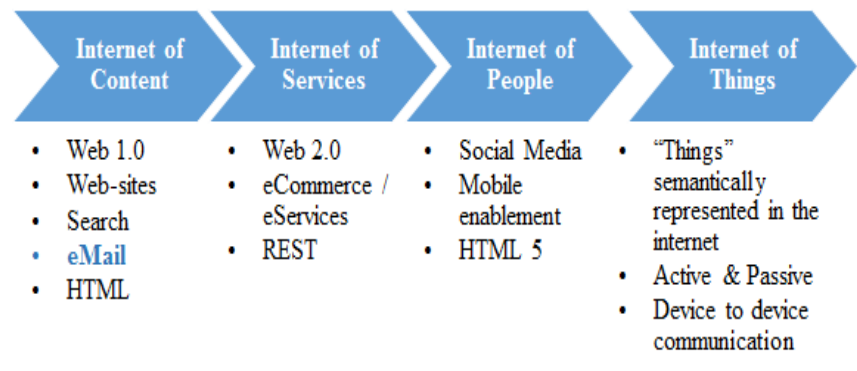

In IoT the notification can be given by various different ways. Such as notification can be given through audio, video, text message, email, software based application. Almost maximum user uses the email for communication. It is standard and professional way to communicate with each other. It's also secure way to communication. To more generalize the all the domain in to one, we have proposed one way to generalize all the domain. This generalized framework and the techniques used to communication are standard. It is being used in all the industry. It is better to use standard way of communication. And lots of features can be provided through this way, so we have proposed IoT based email system to generalize all the domain. Using this technique the notification send by email and text message alert. Automation consist in Internet of Things in itself, and the we provided the manual control over the things which are connected to the node which is connected to the internet. Not only the manual configuration and control is provided but also the summary of events and actions performed on it and much more details are tracked and all logs maintained and can be analyzed using the different data mining techniques which could be used for finding the causes of failure and tells what care should be taken. This way it provides various benefits in all the domains. Definitely the performance of such all domain systems get increases. In the proposed architecture the email is used as a communication medium. There are various clients as shown in architecture. The primary email services are provided to the email client and the IoT serveries also. One of the example is controlling the things from remote location from desktop mobile as remote con-troller etc. The system is completed deployed on the cloud. The different types of database are used to store the sensor as well as the primary email information, all exists on cloud. This system is generic which can be used in all the domains like industry, college, home etc, for automation. There can be multiple number of smart devices (things) under different domains and can be controlled, traced from only one generic application that is IoT based e-mail system. We can store the all the information of events and the action taken on event.

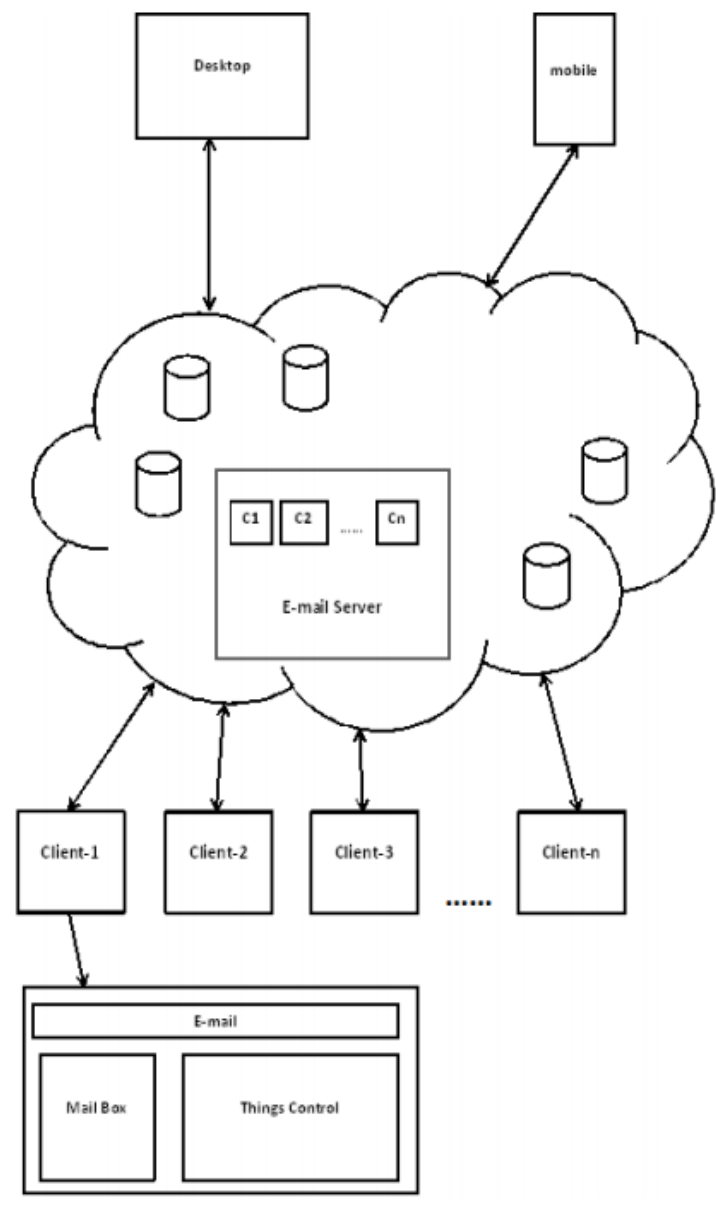

Figure: IoT email-system with cloud

\section{Mathematical Model}

M:= IoT based E-Mail System with cloud

Start:= Human/Things

End:= Alert

$\mathrm{X}:=$ Event, Control, Threshold

$\mathrm{Y}:=$ Action, Notification

Deterministic Data(DD):= Action, Alert, Type-Event

Non-deterministic Data(NDD):= Event-Time

Fmain $:=$ E-mail as communication medium

Ff $\mathrm{r}$ iend $:=$ Cloud

Fmemor y := Emailmem ;MMCmem

Let $\mathrm{A}$ be the set of action

$\mathrm{A}=\mathrm{f} \mathrm{a} 1, \mathrm{a} 2, \mathrm{a} 3, \ldots \ldots \ldots, \mathrm{an}$

$\mathrm{T}$ be the set of things (sensors/object)

connected to internet

$\mathrm{TH}=$ th 1, th 2, th $3, \ldots \ldots . .$, thn

ET be the set of time of occurrence of the event

ET $=$ et 1, et 2, et $3, \ldots \ldots \ldots .$, etn

$\mathrm{E}$ be the set of event

$\mathrm{E}=\mathrm{e} 1, \mathrm{e} 2, \mathrm{e} 3, \ldots \ldots \ldots, \mathrm{en}$

THR be the set of threshold values

THR $=$ thr 1, thr 2, thr $3, \ldots \ldots . . .$, thr $\mathrm{n}$ 


\section{International Journal of Science and Research (IJSR) \\ ISSN (Online): 2319-7064}

Index Copernicus Value (2013): 6.14 | Impact Factor (2015): 6.391

$$
\begin{gathered}
Y=\left(e_{i} \rightarrow a_{i \in A}\right) \wedge\left(\operatorname{thri}_{\mathrm{i}} \in T H R\right) \rightarrow\left(n t_{i} \in N T \wedge \text { Email }_{\text {min } \_ \text {mem }}\right) \\
\text { Where. } \\
a_{i}=\{\text { on, off, read, write }\} \\
\qquad h r_{i} \in\left(V_{k} \vee V_{k} \ldots V_{n-k}:\right. \\
\quad \text { Where, } \\
\mathrm{k}=1,2,3,4 \ldots, \mathrm{n}
\end{gathered}
$$$$
X: \forall_{\theta} \in E
$$

Above mathematical model represents the whole system. Set theory is used to represents the system. As it is represented the entry points are human and things and denoted by symbol $\mathrm{S}$ i.e. starting point. End point is the alert to be sent to the respective user. Event and the threshold value are the input for the system denoted by $\mathrm{X}$ and output is alert or notification denoted by $\mathrm{Y}$. there are deterministic and non-deterministic data. Action, event, type of event are the deterministic data where as event time is the non- deterministic data which cant be predicted. And finally output will be alert will be given to the user in the form of e-mail.

\section{Algorithm}

$\mathrm{E}=$ Set of Events

$\mathrm{D}=$ Set of Devices

$\mathrm{A}=$ Set of Action $\left\{\mathrm{a} 1=0 \mathrm{~N}_{s} \mathrm{a} 2=\mathrm{OFF}_{x} \mathrm{a} 3=\operatorname{Read}_{\mathrm{s}} \mathrm{a} 4=\right.$ Write $x: \forall e_{\mathrm{i}} \in \mathrm{E}$

$$
i=1
$$

for $i=1$ to $D_{s}$

$$
\text { if }\left(\mathrm{d}_{\mathrm{i}(\text { Required_walue })}>\left(\mathrm{th}_{\mathrm{i}} \in \mathrm{THR}\right)\right)
$$

1] $\mathrm{Y}:=\left(\mathrm{e}_{\mathrm{i}} \rightarrow \mathrm{a}_{\mathrm{i}} \in \mathrm{A}\right)$

2] (send_packet(servId, clientId ))

3] (send_notification(mail))

else

return:

\section{Result}

This way we have implemented the IoT based efficient mail system, where we provide the automation and manual control to the user via email system. User can control the home appliances via this email system. If something or devices consuming the electricity unnecessarily the system get OFF automatically and notification will be send to the user via email.

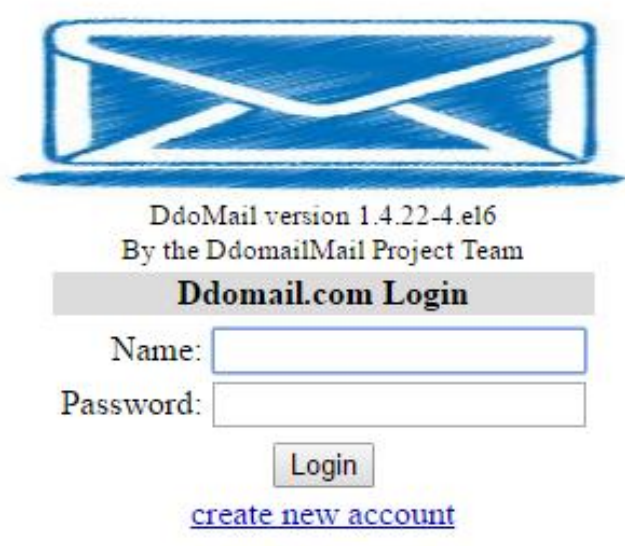

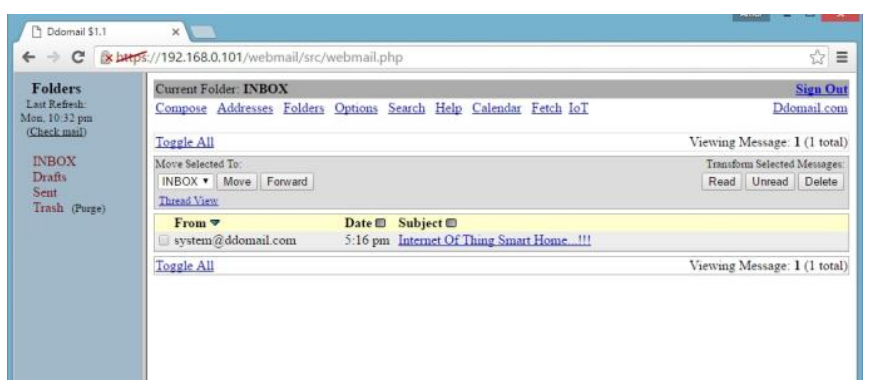

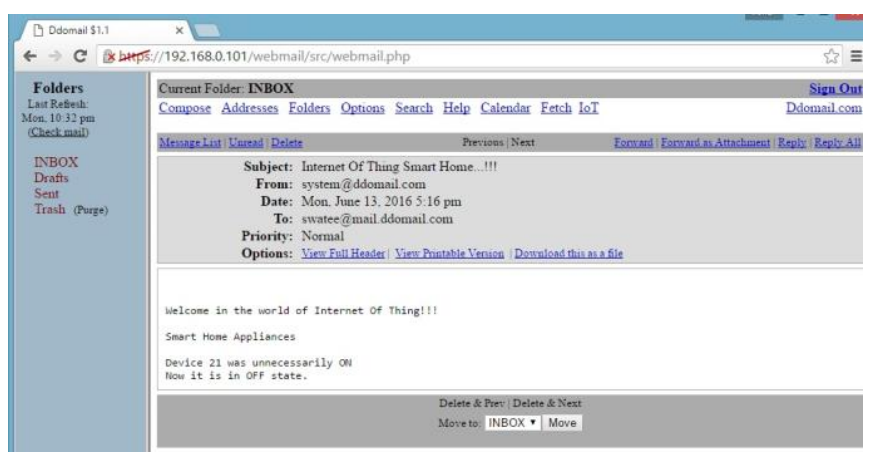

Fig. e-mail notification

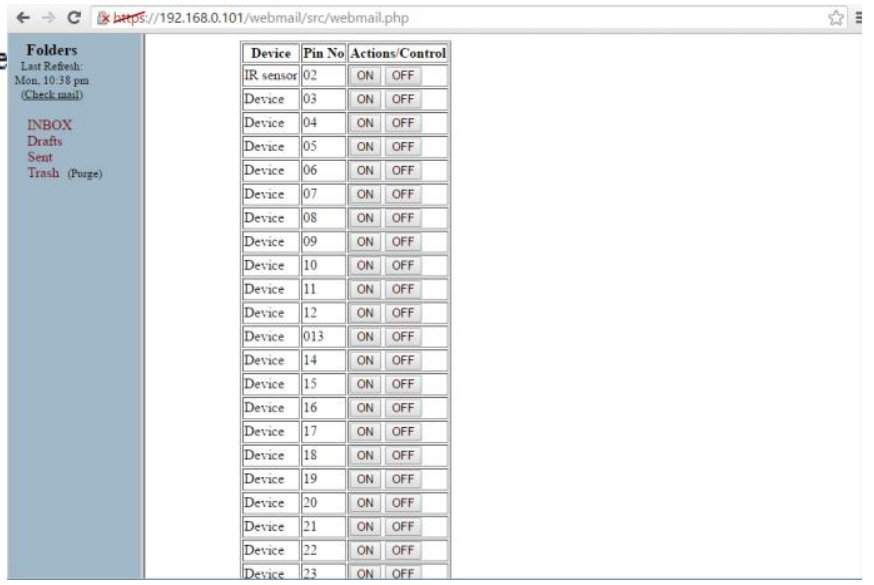

Fig. devices controlling window

\section{Conclusion and Future work}

IoT is growing rapidly almost all the domains are impacting by Internet of Things. Every-things is changing from manual to automate. Internet of Things is itself automization and everything is getting smart work. To making the work efficient and more Internet of Things plays very big role by doing smart work. So to making the almost all the work easy in all the domains IoT is used. And for the secure and efficient communication as well as the alert or notification is the best and efficient way. To generalize and for secure as well as the standard way of communication is e-mail. Even currently almost all system deployed on the cloud for better performance and various benefits of cloud, one of the property is the sharing the resources. It is better to use e-mail as communication medium with cloud.

\section{Acknowledgement}

This work is sponsored by DSM Bhalerao Infotech, Mundhwa- pune. 


\section{International Journal of Science and Research (IJSR) \\ ISSN (Online): 2319-7064}

Index Copernicus Value (2013): 6.14 | Impact Factor (2015): 6.391

\section{References}

[1] S. M. Metev and V. P. Veiko, Laser Assisted Microtechnology, 2nd ed., R. M. Osgood, Jr., Ed. Berlin, Germany: Springer-Verlag, 1998.

[2] Perera, Charith, et al, "A survey on internet of things from industrial market perspective", Access, IEEE 2 (2014): 16601679.

[3] Gazis, Vangelis, et al."Short Paper: IoT: Challenges, Projects,Architectures". Intelligence in Next Generation Networks (ICIN), 2015 18th International Conference on. IEEE, 2015.

[4] TCS ,2015 , Internet of Things Solutions, [online]Available , http://iot-analytics.com/product/iotcompany-ranking-q3q4-2015/

[5] M. Wegmuller, J. P. von der Weid, P. Oberson, and N. Gisin, "High resolution fiber distributed measurements with coherent OFDR," in Proc. ECOC'00, 2000, paper 11.3.4, p. 109.

[6] Perera, Charith, Chi Harold Liu, and Srimal Jayawardena. "The emerging internet of things marketplace from an industrial perspective: a survey."Emerging Topics in Computing, IEEE Transactions on 3.4 (2015): 585-598.

[7] Al-Fuqaha, Ala, et al. "Internet of things: A survey on enabling technologies, protocols, and applications." Communications Surveys \& Tutorials, IEEE17.4 (2015): 2347-2376

[8] K.Kujala (Thursday, 29 Oct, 2015 ), quality hardware list of IoT project s, [online] Available https://thingsee.com/blog/quality-hardware-list-for-youriot-projects.

[9] Kantarci, Burak, and Hussein T. Mouftah. "Sensing services in cloud-centric Internet of Things: A survey, taxonomy and challenges." Communication Workshop (ICCW), 2015 IEEE International Conference on. IEEE, 2015

[10] Salesforce (2016), Introducing salesforce IoT cloud powered by thunder , [online] Available http://www.salesforce.com/in/iot-cloud/

[11]Bosch (2006) ,Internet of Things platform , [online] Available https://www.bosch-si.com/products/bosch-iotsuite/iot-platform/benefits.html

[12] C O'conner (2016) Challenges of Internet of Things , [online] Available http://www.ibm.com/internet-ofthings/learn/what-is-watson-iot/

[13] Amazon (2016) AWS IoT Device SDK, [online] Available https://aws.amazon.com/iot/sdk/

[14] cisco (2015) cisco IoT cloud connect [online] Available http://www.cisco.com/c/en/us/solutions/service provider/iot-cloud-connect/index.html

\section{Author Profile}

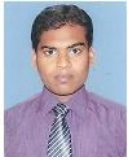

Amol J. Dande $\mathrm{He}$ is post graduate stu-dent in Department of Computer Engineer-ing at STESs Smt. Kashibai Navale Col-lege of Engineering, Pune. He has ob-tained his B.Tech in Computer Engineer-ing from Savitribai Phule Pune Univer-sity, Pune, India. Research Area: Internet of Things.
Shaffi K. Pathan He is having more than 13 year of teaching experience and now currently working as a Asst. Prof. in Smt. Kashibai Navale College of Engineering, Pune for 7 years. $\mathrm{He}$ has worked as alecturer in MIT Engineering College, Au-rangabad for 7 years. Taught the sub-ject like Computer Organization, ComputerGraphics, Operating System, Network and Information Security, Information security and audit manage-ment, Java Programming Language. He has published four research paper in International Journal and eleven research paper in national conference. He can be reached as skpathan@ sinhgad.edu 